\title{
LOS HONGOS MICORRIZÓGENOS ARBUSCULARES DE LA REGIÓN dE NizANDA, OAXACA, MÉXICO
}

\author{
Patricia Guadarrama-Chávez ${ }^{1,4}$, Sara lucía Camargo-Ricalde², Laura Hernández- \\ CUEVAS $^{3}$ y Silvia CASTILLO-ARGÜERO 1
}

\author{
'Departamento de Ecología y Recursos Naturales, Facultad de Ciencias, \\ Universidad Nacional Autónoma de México, México 04510, D.F., México. \\ 2 Departamento de Biología, División de Ciencias Biológicas y de la Salud, \\ Universidad Autónoma Metropolitana-Iztapalapa, Apdo. Postal 55-535, México 09340, D.F., México. \\ ${ }^{3}$ Centro de Investigaciones en Ciencias Biológicas, Laboratorio de Micorrizas, \\ Universidad Autónoma de Tlaxcala, km 10 Carretera Texmelucan-Ixtacuixtla, Tlaxcala 90122, Tlaxcala, México. \\ ${ }^{4}$ Autor para la correspondencia. Correo-e: pgc@fciencias.unam.mx
}

\begin{abstract}
Resumen: Se reportan 25 especies y siete géneros de esporas de hongos micorrizógenos arbusculares (HMA) para la región de Nizanda, Oaxaca, México. Para ello, se tomaron al azar muestras de suelo, en lluvia y secas, incluyendo parcelas de cultivo de maíz, áreas de vegetación secundaria y selva baja caducifolia. Se aislaron las esporas, se identificaron y se montaron macetas de propagación. La familia Glomeraceae aportó 44\% de las especies, seguida de Acaulosporaceae (24\%) y Gigasporaceae (20\%). Se encontraron 13 especies en el maizal, 24 en vegetación secundaria y 12 en selva baja caducifolia. De éstas, Glomus dussi, G. verruculosum, Pacispora scintillans y Scutellospora erythropa son nuevos registros para México. G. constrictum se encontró sólo en el cultivo de maíz, mientras que Acaulospora delicata, A. foveata, A. mellea, A. scrobiculata, Entrophospora infrequens, Gigaspora decipiens, Glomus claroideum, G. fulvum y G. geosporum estuvieron presentes en los tres ambientes estudiados. No se encontraron especies restringidas únicamente a la selva baja caducifolia.
\end{abstract}

Palabras clave: maizal, micorriza arbuscular, riqueza de especies, selva baja caducifolia, vegetación secundaria.

\begin{abstract}
Twenty five species and seven genera of micorrhizal arbuscular fungi (AMF) are reported for the region of Nizanda, Oaxaca, Mexico. To this end, soil samples were taken randomly, during the rainy and the dry seasons, in corn fields, secondary vegetation areas, as well as in primary tropical dry forest. Spores were isolated, identified and propagation pots were set. The family Glomeraceae accounted for $44 \%$ of the species, followed by Acaulosporaceae (24\%) and Gigasporaceae (20\%). In the corn fields 13 species were found, 24 in secondary vegetation, and 12 in the tropical dry forest. Among these, Glomus dussi, G. verruculosum, Pacispora scintillans and Scutellospora erythropa are new records for Mexico. G. constrictum was encountered only in the corn fields, whereas Acaulospora delicata, A. foveata, A. mellea, A. scrobiculata, Entrophospora infrequens, Gigaspora decipiens, Glomus claroideum, G. fulvum and G. geosporum occurred in the three environments. No species were restricted to the tropical dry forest.
\end{abstract}

Key words: arbuscular mycorrhizae, corn field, secondary vegetation, species richness, tropical dry forest.

$\mathbf{L}$ os hongos micorrizógenos arbusculares (HMA) forman una asociación mutualista al colonizar las raíces de las plantas, llamada micorriza arbuscular, la cual solventa los requerimientos de nutrientes y agua de las especies vegetales, con lo que estas últimas aumentan su adecuación. Las plantas, por su parte, le proporcionan al hongo azúcares necesarios para su funcionamiento. Los HMA forman esporas asexuales e hifas cenocíticas capaces de explorar un mayor volumen de suelo que las raíces mismas (Smith y Read, 1997), por lo que son un elemento importante en la estabilización y formación de suelo, al evitar su erosión y ayudar en su rehabilitación (Haselwandter, 2000).

No obstante la importancia de este grupo de hongos, en México se han registrado sólo 44 de las cerca de 200 especies conocidas a nivel mundial. Los registros proceden de 11 entidades federativas y la mayoría de zonas agrícolas. 
En contraste, sólo siete de ellas se reportan de ambientes naturales y únicamente dos, Acaulospora foveata y Glomus clavisporum para el estado de Oaxaca (Varela y Trejo, 2001). Esta información es un fuerte indicativo del gran desconocimiento que existe acerca de la riqueza de especies de hongos micorrizógenos arbusculares en el país y del papel que pueden estar jugando en la dinámica del suelo y en el desarrollo de las plantas con las que se asocian.

Tanto la vegetación primaria como la perturbada de selva baja caducifolia está asociada con HMA (Allen et al., 1998; Allen et al., 2003), así como la mayoría de las plantas cultivadas (Munyanziza et al., 1997; Douds y Jhonson, 2003). La asociación de éstas con los HMA puede solventar sus requerimientos de fósforo $(\mathrm{P})$, como ocurre con el maíz (Zea mays) (Wright et al., 2005). Cuando los terrenos de cultivo son abandonados, la composición de los HMA y su diversidad funcional pueden influir en la velocidad de regeneración de las comunidades vegetales, ya que su presencia en las raíces de las plantas hospederas facilita la exploración y toma de nutrimentos del suelo (Cui y Caldwell, 1996), con repercusiones positivas en el crecimiento y la supervivencia. Aun así, son escasos los estudios que describen las especies de HMA presentes en comunidades naturales y en terrenos de cultivo abandonados. Por ello, el objetivo de este estudio fue proporcionar un panorama general de la riqueza de especies de HMA en la selva baja caducifolia, en las áreas de vegetación secundaria producida por el abandono de campos de cultivo, y en maizales originados por la tala de la vegetación natural en una región tropical estacionalmente seca del sur de México.

\section{Materiales y métodos}

El trabajo se llevó a cabo en la región de Nizanda, Oaxaca, México (16³9' N y 9500' O). Las muestras de suelo se recolectaron en un terreno cultivado con maíz por un año y cosechado recientemente, en tres parcelas de vegetación secundaria de selva baja caducifolia originadas a partir del sistema agrícola de roza-tumba-quema con tres, cinco y diez años de abandono (Lebrija-Trejos et al., en prensa), y en un área de vegetación natural. En los sitios donde hubo actividad agrícola, ésta fue de tipo tradicional, con uso de azadón, sin fe rtilizantes y con corta y quema de la vegetación natural en época de secas. Posteriormente se sembró maíz por una única vez, se cosechó y se abandonó la parcela.

El clima en la región es de tipo Awo (w) igw", es decir, caliente subhúmedo con lluvias en verano (García, 2004) y con una marcada estacionalidad, distinguiéndose una estación de sequía (noviembre a abril) y una lluviosa (mayo a octubre) (Anónimo, 1984a, b). Los tipos de suelo predominantes son litosoles y como suelos secundarios hay zonas de feozems háplicos y regosoles éutricos, ambos con estructuras medias (Anónimo, 1981). Waltheria indica L. es la especie dominante en la parcela de maíz recientemente abandonada; Mimosa acantholoba (Humb. et Bonpl. ex Willd.) Poir var. eurycarpa B.L.Rob., Melochia tomentosa L., Senna holwayana (Rose) H.S.Irwin et Barn eby y Mimosa tenuiflora (Willd.) Poir son las especies más abundantes en los sitios de vegetación secundaria, mientras que Justicia caudata A.Gray lo es en la selva natural (LebrijaTrejos, 2004; Lebrija-Trejos et al., en prensa).

Muestreo de suelo y establecimiento de cultivos trampa. El muestreo se realizó en octubre de 2005 y mayo de 2006. En cada sitio de estudio se recolectaron tres muestras de suelo de $2 \mathrm{~kg}$ cada una en puntos marcados al azar dentro del sitio y se integró una muestra mixta completamente homogenizada. Antes de tomar la muestra de suelo se eliminó la materia orgánica de los $15 \mathrm{~cm}$ superiores de suelo. Se llevó a cabo el aislamiento de las esporas de HMA por el método de tamizado húmedo (Gerdemann y Nicolson, 1963) y flotación en gradientes de sacarosa (Daniels y Skipper, 1982, modificado por Brundett et al., 1996), de tres submuestras de $50 \mathrm{~g}$ por sitio y por fecha de muestreo. Al mismo tiempo, se colocaron $300 \mathrm{~g}$ de suelo de cada parcela en macetas ( $500 \mathrm{~mL}$ de capacidad) con semillas de sorgo (Sorgum vulgare L.), que fue seleccionado como planta trampa debido a su temprana colonización micorrízica y alta supervivencia. A los seis meses después de la siembra, se tomaron tres muestras de $50 \mathrm{~g}$ por maceta, se extrajeron las esporas y se elaboraron preparaciones permanentes para corroborar la identidad de las especies de HMA encontradas en las muestras de campo.

Identificación de especies de HMA. Las esporas aisladas de las muestras de campo y de los cultivos trampa se midieron, se cuantificaron, y se observó su consistencia, arreglo y ornamentación, así como la reacción con Melzer de los estratos de la pared de las esporas. La determinación de las especies se realizó considerando descripciones especializadas de las especies de los diferentes géneros y la información del International Culture Collection of (Vesicular) Arbuscular Mycorrhizal Fungi (INVAM), disponible a través de la Internet (invam.caf.wvu.edu/myc_info/ taxonomy). Las preparaciones permanentes de esporas de HMA se etiquetaron con los datos de herbario correspondientes y se depositaron en el herbario TLXM de la Universidad Autónoma de Tlaxcala, donde se encuentran disponibles para consulta.

\section{Resultados}

Se registra ron 25 especies de hongos micorrizógenos arbusculares (HMA) pertenecientes a seis familias y siete géneros (apéndice 1). La familia Glomeraceae aportó 44\% de las especies, seguida de Acaulosporaceae y Gigasporaceae, con $24 \%$ y $20 \%$, respectivamente. En los 


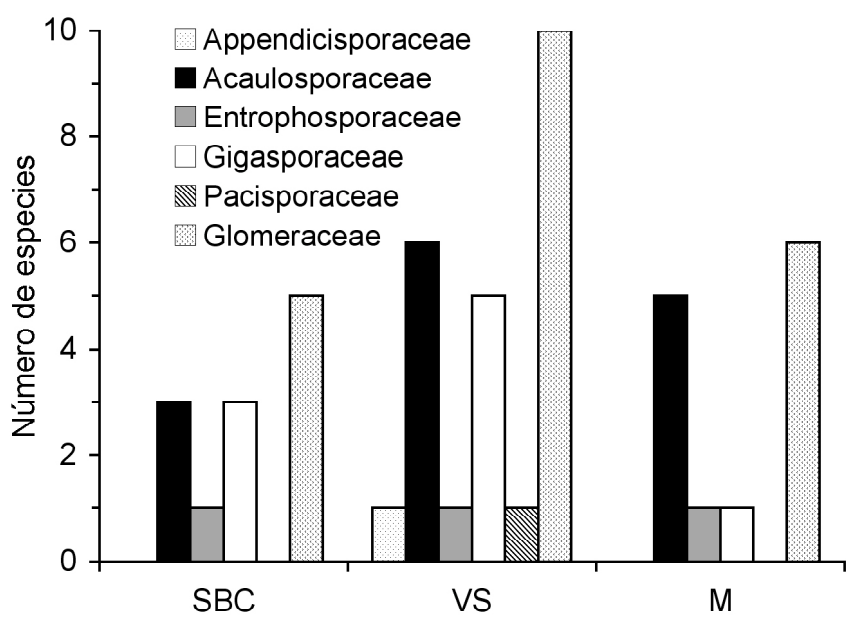

Figura 1. Número de especies encontradas por familia en un área de selva baja caducifolia (SBC), en vegetación secundaria (VS) y en un terreno cultivado con maíz (M), en la región de Nizanda, Oaxaca, México.

sitios de vegetación secundaria se encontraron representantes de todas las familias, mientras que en la zona de selva baja caducifolia y en el maizal estuvieron ausentes las familias Appendicisporaceae y Pacisporaceae (figura 1). De las especies registradas, Acaulospora delicata, A. fove ata, A. mellea, A. scrobiculata, Entrophospora infrequens, Gigaspora decipiens, Glomus claroideum, G. fulvum, G. geosporum estuvieron presentes en el maizal, vegetación secundaria y selva baja caducifolia. Glomus constrictum, Appendicispora appendicula, Acaulospora morrowae, Scutellospora dipurpurascens, S. erythropa, Pacispora scintillans, G. aggregatum, G. microaggregatum, G. sinuo sum y G. verruculosum fueron observados sólo en un sitio, la primera sólo en el cultivo de maíz y las restantes en vegetación secundaria.

Cuatro de las especies encontradas, $P$. scintillans [sinónimo Gerdemania scintillans (Walker y Schüßler, 2004), sinónimo Glomus scintillans (Oehl y Sieverding, 2004)], G. dussi (sinónimo Sclerocystis dussi (Almeida y Schenck, 1990), Glomus verruculosum y Scutellospora erythropa se reportan aquí por vez primera para México. Acaulospora foveata, S. dipurpurascens, G. fulvum y G. geosporum se han reportado sólo para agrosistemas, mientras que $A$. morrowae, E. infrequens, G. aggregatum, G. tenebrosum y las cuatro especies que se reportan para México por primera vez sólo se habían sido registradas en ecosistemas naturales. De estas últimas, sólo G. tenebrosum había sido encontrada en selva baja caducifolia, en Jalisco, México (Varela y Trejo, 2001). El resto de las especies (12) cuentan con registros tanto de ecosistemas naturales como de agrosistemas.

La identificación de las especies se basó en esporas extraídas de suelos de campo de los tres sitios muestreados y fue corroborada con esporas procedentes de las macetas de propagación preparadas con los mismos suelos. De cinco especies, A. morrowae, S. erythropa, P. scintillans, Gigaspora decipiens y $E$. infrequens se encontraron menos de 10 esporas en $100 \mathrm{~g}$ de suelo seco en las macetas de propagación. Glomus clavisporum y G. sinuosum no produjeron esporas en dichas macetas. Del resto de las especies se encontraron esporas tanto en el suelo de campo como en las macetas de propagación.

\section{Discusión}

Los parches de vegetación secundaria, resultantes del abandono de campos agrícolas donde se realizó este estudio, tuvieron una riqueza de 24 especies de esporas de hongos micorrizógenos arbusculares (HMA); en la parcela de maíz la riqueza fue de 13 especies y en la vegetación natural de selva baja caducifolia fue de 12 . Aunque se ha señalado que la actividad agrícola disminuye la riqueza de HMA (Muthukumar y Udaiyan, 2000), nuestros resultados no apoyan esta afirmación, ya que nosotros reportamos valores similares para el cultivo de maíz y la vegetación natural. En el sitio de selva baja caducifolia encontramos una riqueza menor que las 15 especies citadas por Allen et al. (1998) para Chamela, Jalisco, México. Estos valores no reflejan la relación entre la diversidad vegetal y la de HMA (van der Heijden et al., 1998a, b), quizá como consecuencia de la pérdida de latencia de las esporas debido a la sequía extrema. Por lo tanto, se hace necesario realizar otros estudios, por ejemplo incluyendo los fragmentos de raíces secas latentes, para evaluar otros tipos de inóculo (Allen et al., 1998).

En comunidades perturbadas se ha observado una variación en la dominancia de algunos géneros de HMA, ya que éstos presentan diferentes estrategias de colonización después de ocurrido un disturbio. El género Glomus ha sido señalado por su incidencia en zonas agrícolas con una alta intensidad de manejo del suelo (Mathimaran et al., 2005), y en particular $G$. aggregatum está reportada en sitios recientemente abandonados, aunque pierde dominancia a lo largo del proceso sucesional (Johnson et al., 1991). En este trabajo sólo se presentó en la parcela de 10 años de abandono. Acaulospora foveata ha sido encontrada en pastizales tropicales en Neguev, Costa Rica, en proporcion de 800 esporas de A. foveata por 600 esporas de Glomus (Picone, 2000). En este estudio, Glomus y Acaulospora se presentaron en las parcelas tanto recientemente abandonadas como en la de vegetación natural, lo que indica que son géneros generalistas y que tienen una alta tolerancia a la perturbación (Boddington y Dodd, 2000). Al aumentar la diversidad vegetal, aumenta la esporulación de los géneros Gigaspora y Scutellospora (Burrows y Pfleger, 2002), los cuales se presentaron predominantemente en los sitios con 
vegetación secundaria. En particular, Gigaspora gigantea se encontró en las parcelas con mayor tiempo de abandono y en el sitio de selva, lo cual sugiere que es una especie poco tolerante a los disturbios, mientras que las especies Glomus geosporum y G. claroideum pueden considerarse "generalistas", ya que estuvieron presentes en todas las parcelas. Oehl et al. (2003) reconocieron a G. geosporum como una especie generalista en parcelas agrícolas y pastizales, lo que concuerda con lo encontrado en este estudio. Por el contrario, las especies presentes en las parcelas más jóvenes y que pueden considerarse "tolerantes a la perturbación" debido a su mayor incidencia en sitios perturbados son A. foveata, S. erythropa, E. infrequens, G. aggregatum y G. constrictum.

Este tipo de estudios son básicos para conocer, en un principio, la biodiversidad de HMA presentes en México, y posteriormente para poder llevar a cabo programas de restauración ambiental con especies nativas, no sólo vegetales, sino de los HMA con los que se asocian y que son importantes en su adecuación. Esto puede aumentar las probabilidades de éxito de estos programas al utilizar especies adaptadas a las condiciones ambientales de regiones específicas del país.

\section{Agradecimientos}

Yuriana Martínez Orea, Diego Olivera, Oswaldo Núñez Castillo, Irene Sánchez Gallén y Eduardo Pérez García colaboraron en el trabajo de campo y de laboratorio. Mayra Gavito, Dora Trejo y Jorge Meave proporcionaron valiosos comentarios sobre una versión anterior del manuscrito. El estudio fue financiado por el proyecto PAPIIT IN221503 (Universidad Nacional Autónoma de México).

\section{Literatura citada}

Allen E.B., Allen M.F., Egerton-Warburton L., Corkidi L. y Gómez-Pompa A. 2003. Impacts of early- and late- seral mycorrhizae during restoration in seasonal tropical forest, Mexico. Ecological Applications 13:1701-1717.

Allen E.B., Rincón E., Allen M.F., Pérez-Jiménez A. y Huante P. 1998. Disturbance and seasonal dynamics of mycorrhizae in a tropical deciduous forest in Mexico. Biotropica 30:261-274.

Almeida R.T. y Schenck N.C. 1990. A revision of the genus Sclerocystis (Glomaceae, Glomales). Mycologia 82:703-714.

Anónimo. 1981. Atlas Nacional del Medio Físico. Secretaría de Programación y Presupuesto, México, D.F.

Anónimo. 1984a. Carta de efectos climáticos regionales mayooctubre. Juchitán E15-10 D15-1, escala 1:250,000. Secretaría de Programación y Presupuesto, México, D.F.

Anónimo. 1984b. Carta de efectos climáticos regionales noviembre-abril. Juchitán E15-10 D15-1, escala 1:250,000. Secretaría de Programación y Presupuesto, México, D.F.

Boddington C.L. y Dodd J.C. 2000. The effect of agricultural practices on the development of indigenous arbuscular mycorr- hizal fungi. I. Field studies in an Indonesian ultisol. Plant and Soil 218:137-144.

Brundett M., Bougher N., Dell B., Grove T. y Malajczuk N. 1996. Working with Mycorrhizas in Forestry and Agriculture. Australian Centre for International Agricultural Research, Monograph, Canberra.

Burrows R.L. y Pfleger F.L. 2002. Arbuscular mycorrhizal fungi respond to increasing plant diversity. Canadian Journal of Botany 80:120-130.

Carrillo-Sánchez L., Barredo-Pool F., Varela L., Arce-Montoya M., Orellana R. 1998. Estudio de la asociación micorrízica en tres especies de palmeras nativas de la península de Yucatán. En: Zulueta R.R., Escalona M.A. y Trejo D. Eds. Avances de la Investigación Micorrízica en México, pp. 77-84, Universidad Veracruzana, Xalapa.

Carrillo-Sánchez L., Varela L., y Orellana R. 2000. Variación estacional en la densidad de esporas de hongos micorrizógenos arbusculares y en el porcentaje de colonización micorrízica de tres palmeras Yucatenses. En: Alarcón A. y Ferrera-Cerrato R. Eds. Ecología, Fisiología y Biotecnología de la Micorriza Arbuscular, pp. 39-45, Colegio de Postgraduados, Mundi Prensa, Montecillo, Estado de México.

Cui M. y Caldwell M.M. 1996. Facilitation of plant phosphate acquisition by arbuscular mycorrhizas from enriched soil patches. New Phytologist 133:453-460.

Daniels B.A. y Skipper H.D. 1982. Methods for the recovery and quantitative estimation of propagules from soil. En: Schenck N.C. Ed. Methods and Principles of Mycorrhizal Research, pp. 29-35, American Phytopathological Society, St. Paul, Minessotta.

Douds Jr D.D. y Johnson N.C. 2003. Contributions of arbuscular mycorrhizas to soil biological fertility. En: Abbott L.K. y Murphy D.V. Eds. Soil Biological Fertility. A Key to Sustainable Land Use in Agriculture, pp. 129-162, Kluwer, Dordrecht, Holanda.

García E. 2004. Modificaciones al Sistema de Clasificación Climática de Köppen. 5a ed., Universidad Nacional Autónoma de México, México, D.F.

Gerdemann J.W. y Nicolson T.H. 1963. Spores of mycorrhizal endogone species extracted from soil by wet sieving and decanting. Transactions of the British Mycological Society 46:235244.

Guadarrama P. y Álvarez-Sánchez F.J. 1999. Abundance of arbuscular mycorrhizal fungi spores in different environments in a tropical rain forest, Veracruz, Mexico. Mycorrhiza 8:267-270.

Haselwandter K. 2000. Soil micro-organisms, mycorrhiza, and restoration ecology. En: Urbanska K.M., Webb N.R. y Edwards P.J. (eds.). Restoration Ecology and Sustainable Development, pp 65-80, Cambridge University Press, Cambridge.

Hernández-Cuevas L., Castillo-Argüero S., Guadarrama-Chávez P., Martínez-Orea Y., Romero-Romero M.A. y Sánchez-Gallén I. 2003. Hongos Micorrizógenos Arbusculares del Pedregal de San Ángel. Las Prensas de Ciencias, Universidad Nacional Autónoma de México, México, D.F.

Johnson N.C., Zak D.R., Tilman D. y Pfleger F.L. 1991. Dynamics of vesicular-arbuscular mycorrhizae during old field succession. Oecologia 86:349-358.

Lebrija-Trejos E.E. 2004. Secondary succession in a tropical dry forest of southern Mexico. Tesis de Maestría, Universidad de Wageningen, Wageningen, Holanda, 68 pp. 
Lebrija-Trejos E.E., Bongers F., Pérez-García E.A. y Meave J.A. En prensa. Successional change and recovery rates in a secondary tropical dry forest. Biotropica.

Mathimaran N., Ruh R., Vullioud P., Frossard E. y Jansa J. 2005. Glomus intraradices dominates arbuscular mycorrhizal communities in a heavy textured agricultural soil. Mycorrhiza 16:61-66.

Munyanziza E., Kehri H.K. y Bagyaraj D.J. 1997. Agricultural intensification, soil biodiversity and agro-ecosystem function in the tropics: the role of mycorrhiza in crops and trees. Applied Soil Ecology 6:77-85.

Muthukumar T. y Udaiyan K. 2000. Arbuscular micorrizas of plants growing in the Western Ghats region, Southern India. Mycorrhiza 9:297-313.

Oehl F. y Sieverding E. 2004. Pacispora, a new vesicular arbuscular mycorrhizal fungal genus in the Glomeromycetes. Journal of Applied Botany 78:72-82.

Oehl F., Sieverding E., Ineichen K., Mäder P., Boller T. y Wiemken A. 2003. Impact of land use intensity on the species diversity of arbuscular mycorrhizal fungi in agroecosystems of central Europe. Applied and Environmental Microbiology 69:2816-2824.

Pezzani F., Guevara R., Hernández-Cuevas L. y Montaña C. En prensa. Mycorrhizal interactions in Mapimí Biosphere Reserve: arbuscular mycorrhizal fungi associated with grasses from the Chihuahuan Desert. En: Montaño N., Camargo-Ricalde S.L., García-Sánchez R. y Monroy-Ata A. comps. Arbuscular Mycorrhizae in Arid and Semiarid Environments, Mundi Prensa, México, D.F.

Recibido: 23 de marzo de 2007

Aceptado: 6 de octubre de 2007
Picone C. 2000. Diversity and abundance of arbuscular mycorrhizal fungus spores in tropical forest and pasture. Biotropica 32:734-750.

Ramírez-Gerardo M., Álvarez-Sánchez J., Guadarrama P. y Sánchez-Gallén I. 1997. Estudio de hongos micorrizógenos arbusculares bajo árboles remanentes en un pastizal tropical. Boletín de la Sociedad Botánica de México 61:15-20.

Smith S.E. y Read D.J. 1997. Mycorrhizal Symbiosis. Academic Press, San Diego.

van der Heijden M.G.A., Boller T., Wiemken A. y Sanders I.R. 1998a. Different arbuscular mycorrhizal fungal species are potential determinants of plant community structure. Ecology 79:2082-2091.

van der Heijden M.G.A., Klironomos J.N., Ursic M., Moutoglis P., Streitwolf-Engel R., Boller T., Wiemken A. y Sanders I.R. 1998b. Mycorrhizal fungal diversity determines plant biodiversity, ecosystem variability and productivity. Nature 396:69-72.

Varela L. y Trejo D. 2001. Los hongos micorrizógenos arbusculares como componentes de la biodiversidad del suelo en México. Acta Zoologica Mexicana (n.s.). Número especial 1:39-51.

Walker C. y Schüßler A. 2004. Nomenclatural clarifications and new taxa in the Glomeromycota. Mycological Research 108:981-982.

Wright D.P., Scholes J.D., Read D. y Rolfe S.A. 2005. European and African maize cultivars differ in their physiological and molecular responses to mycorrhizal infection. New Phytologist 167:881-896. 
Apéndice 1. Listado de especies de hongos micorrizógenos arbusculares (HMA) encontradas en cultivo de maíz (M), vegetación secundaria (VS) y selva baja caducifolia (SBC) en la región de Nizanda, Oaxaca, México

\begin{tabular}{lll}
\hline Especie de HMA & \multicolumn{2}{c}{ Sitio de estudio } \\
\cline { 2 - 4 } & Reporte en México \\
\hline
\end{tabular}

\section{ARCHAEOSPORALES}

\section{APPENDICISPORACEAE}

Appendicispora appendicula (Spain, Sieverd. et N.C.Schenck) Spain, Oehl et Sieverd.

\section{DIVERSISPORALES}

ACAULOSPORACEAE

Acaulospora delicata Walker, Pfeiffer et Bloss

A. foveata Trappe et Janos

A. laevis Gerdemann et Trappe

A. mellea Spain et Schenck

A. morrowae Spain et Schenck

A. scrobiculata Trappe

\section{ENTROPHOSPORACEAE}

Entrophospora infrequens (Hall) Ames et Schneider

\section{GIGASPORACEAE}

Gigaspora decipiens Hall et Abbott

G. gigantea Nicolson et Gerdemann

Scutellospora dipurpurascens Morton et Koske

S. erythropa (Koske et Walker) Walker et Sanders

S. pellucida (Nicol. et Schenck) Walker et Sanders

\section{PACISPORACEAE}

Pacispora scintillans (S.L.Rose et Trappe) Sieverd.

et Oehl ex C. Walker, Vetsberg et Schuessler

\section{GLOMERALES}

\section{GLOMERACEAE}

Glomus aggregatum Schenck et Smith emend. Koske

G. claroideum Schenck et Smith
$X$

$X$

$X$

$\begin{array}{llll}X & X & X & \text { Varela y Trejo (2001) } \\ & & \text { Pezzani et al. (en prensa) } \\ \text { X } & X & \text { Varela y Trejo (2001) } \\ \text { X } & \text { X } & & \text { Varela y Trejo (2001) }\end{array}$

Hernández-Cuevas et al. (2003)

Pezzani et al. (en prensa)

X

Varela y Trejo (2001)

Hernández-Cuevas et al. (2003)

Pezzani et al. (en prensa)

X Ramírez-Gerardo et al. (1997)

Carrillo et al. (1998), (2000)

Guadarrama y Álvarez-Sánchez (1999)

Varela y Trejo (2001)

Hernández-Cuevas et al. (2003)

X Varela y Trejo (2001)

Hernández-Cuevas et al. (2003)

Pezzani et al. (en prensa)

$X$

$X$

$X$

$X$

Pezzani et al. (en prensa)

Varela y Trejo (2001)

Hernández-Cuevas et al. (2003)

$x$

$x$

$X$

$X$

Varela y Trejo (2001)

Primer reporte en México

Varela y Trejo (2001)

Hernández-Cuevas et al. (2003)

X

Primer reporte en México
Hernández-Cuevas et al. (2003)

Pezzani et al. (en prensa)

Varela y Trejo (2001)

Hernández-Cuevas et al. (2003)

Pezzani et al. (en prensa) 
LOS HONGOS MICORRIZÓGENOS DE NIZANDA, OAXACA

\begin{tabular}{|c|c|c|c|c|}
\hline \multirow[t]{2}{*}{ Especie de HMA } & \multicolumn{3}{|c|}{ Sitio de estudio } & \multirow[t]{2}{*}{ Reporte en México } \\
\hline & $\bar{M}$ & VS & SBC & \\
\hline G. clavisporum (Trappe) Almeida et Schenck & & $x$ & $x$ & Varela y Trejo $(2001)^{* *}$ \\
\hline \multirow{2}{*}{ G. constrictum Trappe } & $x$ & & & Varela y Trejo (2001) \\
\hline & & & & Pezzani et al. (en prensa) \\
\hline G. dussi (Thaxter) Almeida et Schenck & $x$ & $\mathrm{X}$ & & Primer reporte en México \\
\hline G. fulvum (Berk. et Broome) Trappe et Gerd. & $x$ & $\mathrm{x}$ & $\mathrm{x}$ & Varela y Trejo (2001) \\
\hline \multirow[t]{4}{*}{ G. geosporum (Nicolson et Gerdemann) Walker } & $x$ & $x$ & $x$ & Carrillo et al. (1998), (2000) \\
\hline & & & & Varela y Trejo (2001) \\
\hline & & & & Hernández-Cuevas et al. (2003) \\
\hline & & & & Pezzani et al. (en prensa) \\
\hline \multirow[t]{3}{*}{ G. microaggregatum Koske, Gemma et Olexia } & & $x$ & & Varela y Trejo (2001) \\
\hline & & & & Hernández-Cuevas et al. (2003) \\
\hline & & & & Pezzani et al. (en prensa) \\
\hline G. tenebrosum (Thaxter) Berch & $x$ & $x$ & $x$ & Varela y Trejo (2001) \\
\hline G. sinuosum (Gerd. et Bakshi) Almeida et Schenck & & $x$ & & Varela y Trejo (2001) \\
\hline G. verruculosum Blaszkowski et Tadych & & $x$ & & Primer reporte en México \\
\hline
\end{tabular}

* Especie citada como Acaulospora appendicula

**Especie citada como Sclerocystis clavispora 